\title{
Beta-Carotin - ein gefährlicher Mikronährstoff für Raucher?
}

Anfang 2006 hat das Bundesinstitut für Arzneimittel und Medizinprodukte (BfArM) einen Bescheid über Beta-Carotin-haltige Arzneimittel veröffentlicht, nachdem bei Beta-Carotin-Dosierungen von mehr als 20 mg/Tag seit 1.5.2006 im Abschnitt "Gegenanzeigen " folgender Hinweis aufgenommen werden muss:

"(Arzneimittelname) darf von starken Rauchern (20 und mehr Zigaretten/Tag) nicht eingenommen

Die Sinnhaftigkeit einer werden. In klinischen Studien Untergrenze von war das Risiko für das Auftreten von Lungenkrebserkrankungen $2 \mathrm{mg}$ ist zu hinterfragen bei Rauchern erhöht, wenn zusätzlich zur normalen Ernährung täglich 20 mg Beta-Carotin über einen längeren Zeitraum (bis 24 Monate) eingenommen wurden."

Arzneimittel mit einem Beta-Carotin-Gehalt von 2 bis 20 mg/Tag müssen die vorgenannte Kennzeichnung zwar nicht im Abschnitt "Gegenanzeigen" enthalten, jedoch einen weitgehend ähnlichen Text in den Abschnitt "Besondere Warn- und Vorsichtsmaßnahmen für die Anwendung " aufnehmen. Nur Arzneimittel, die weniger als $2 \mathrm{mg}$ Beta-Carotin/Tag zuführen, müssen solche Hinweise nicht aufnehmen.

Grundlage dieser Anwendungsbeschränkungen sind zwei Interventionsstudien (ATBC und CARET). In diesen Studien war die Lungenkrebsinzidenz und -mortalität bei langjährigen starken Rauchern nach hoch dosierter Supplementierung mit Beta-Carotin (20 mg/Tag beziehungsweise 30 mg/Tag) im Vergleich zu einer Placebogruppe signifikant erhöht. In einer Reihe von weiteren Interventionsstudien an verschiedenen Kollektiven wie Nichtrauchern, ehemaligen Rauchern und Rauchern, wurde hingegen unter BetaCarotin-Gabe kein erhöhtes Risiko für Lungenkrebs beobachtet (Physicians' Health Study, MRC/BHF-Heart Protection Study Collaborative Group, Womens' Health Study, AREDS Research Group, REACT).

Trotz uneinheitlicher Studienlage erscheinen im vorrangigen Interesse des Verbraucherschutzes Warnhinweise bezüglich der Gabe von Beta-Carotin in hohen Dosierungen (Dosierungen, die den geschätz- ten Tagesbedarf deutlich überschreiten) bei Rauchern als nachvollziehbar.

Anders verhält es sich mit der Untergrenze von $2 \mathrm{mg}$, die bereits verpflichtende Warnhinweise erfordert. Beta-Carotin ist nicht nur als Provitamin A ein wichtiger Mikronährstoff, sondern es dürften ihm eigenständige physiologische Funktionen im Rahmen antioxidativer Schutzmechanismen zukommen. Vor allem bei Vegetariern und Veganern ist Beta-Carotin eine praktisch unersetzliche Vitamin-A-Quelle und angereicherte Nahrungsmittel, Nahrungsergänzungen und Arzneimittel können für eine ausreichende BetaCarotin-Versorgung sinnvoll und zum Teil sogar notwendig sein. Ähnliche Überlegungen gelten für Risikogruppen unter einseitiger Ernährung oder Reduktionsdiäten sowie bei erhöhten Bedarfssituationen. Die gemeinsame Kommission der deutschen, schweizerischen und österreichischen Gesellschaften für Ernährung gibt hinsichtlich der notwendigen Zufuhr von Beta-Carotin über die Nahrung einen Schätzwertbereich von 2 bis $4 \mathrm{mg}$ pro Tag an. Britische Empfehlungen nennen für die Supplementierung mit Beta-Carotin einen "Safe Upper Intake Level« von 7 mg/Tag.

Betrachtet man die Beta-Carotin-Konzentrationen in Nahrungsmitteln, so werden über $100 \mathrm{~g}$ Karotten im Durchschnitt $7 \mathrm{mg}$ Beta-Carotin und über jeweils $100 \mathrm{~g}$ rote Beete oder Grünkohl zirka 5 mg BetaCarotin aufgenommen. Die angegebene Menge von $2 \mathrm{mg}$ Beta-Carotin/Tag kann damit bei entsprechender Ernährung leicht überschritten werden (auch unter Berücksichtigung unterschiedlicher Bioverfügbarkeiten von Beta-Carotin in Vegetabilien im Vergleich zu synthetischem Beta-Carotin). Dies gilt nicht zuletzt dann, wenn man aktuellen Ernährungsempfehlungen folgt und fünf Portionen Gemüse und Obst pro Tag verzehrt. Es stellt sich also die Frage, wann mit den ersten Warnhinweisen für Raucher hinsichtlich des Verzehrs bestimmter Beta-Carotin-reicher Gemüseoder Obstsorten gerechnet werden muss.

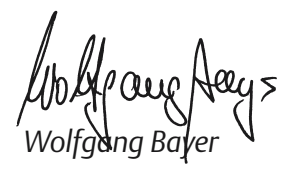

